\title{
Autonomia e Exercício de Direitos na Experiência da Gestão Autônoma da Medicação
}

\author{
Analice Palombini ${ }^{1}$ \\ Marciana Zambillo ${ }^{1}$ \\ ${ }^{1}$ Universidade Federal do Rio Grande do Sul, RS, Brasil. \\ ${ }^{1}$ Universidade Federal do Rio Grande do Sul, RS, Brasil. \\ Vera Pasini \\ Sandra Zanatta Guerra ${ }^{1}$ \\ ${ }^{1}$ Universidade Federal do Rio Grande do Sul, RS, Brasil. \\ ${ }^{1}$ Universidade Federal do Rio Grande do Sul, RS, Brasil. \\ Lívia Zanchet ${ }^{1}$ \\ Antonio B. Oliveira Neto ${ }^{1}$ \\ ${ }^{1}$ Universidade Federal do Rio Grande do Sul, RS, Brasil. \\ ${ }^{1}$ Universidade Federal do Rio Grande do Sul, RS, Brasil. \\ Julia Bongiovanni ${ }^{1}$ \\ Letícia Presser Ehlers ${ }^{1}$ \\ ${ }^{1}$ Universidade Federal do Rio Grande do Sul, RS, Brasil. \\ ${ }^{1}$ Universidade Federal do Rio Grande do Sul, RS, Brasil.
}

Resumo: O artigo investigou os efeitos de uso da versão brasileira do Guia da Gestão Autônoma da Medicação (GAM-BR) em grupos de intervenção em serviços públicos saúde mental. Objetiva-se analisar narrativas de usuários, psiquiatras e demais profissionais a partir da relação de cada um deles com a prescrição medicamentosa, mote do trabalho como Guia. Participaram dapesquisa três CAPS do sul do país, integrantes da pesquisa multicêntrica GAM-BR. O áudio das falas produzidas nos grupos focais e nas entrevistas foi gravado, transcrito e transformado em narrativas por meio da extração dos núcleos argumentais. Os resultados apontam para a ampliação da concepção de autonomia e maior reconhecimento dos direitos dos usuários. Sugere, porém, dificuldades no exercício desses direitos, especialmente com relação ao tratamento medicamentoso, visto como condição para manutenção do vínculo com os serviços. Ressalta a importância de maior reflexão, tendo em vista a manutenção da lógica da escolha, privatizante e individualista, em detrimento da lógica do cuidado que valoriza o trabalho em rede e a corresponsabilização.

Palavras-chave: Saúde Mental, Narrativas, Autonomia, Cidadania, Medicação.

\section{Autonomy and Rights Exercise in Autonomous Management of Medication Experience}

\begin{abstract}
This article analyzed the effects of using the Brazilian version of the Autonomous Medication Management Guide (GAM-BR) in intervention groups in mental health services. Users, psychiatrists and other professionals' narratives were verified to check the relationship of each one with the prescription, the main principle of the guide. Three CAPS (mental health care services) from the south of the country have participated in this research, all members of the multicentric research GAM-BR. The material was audio-recorded, transcribed and transformed in narratives. The results show increase of the conception of autonomy and wider acknowledgment of users' rights. A broadening concept of users' autonomy and a greater recognition of their rights was observed. However, issues were found in the exercise of rights, especially regarding drug treatment, which was seen as a condition for their bond maintenance with services. Further reflection on this subject is emphasized, owing to maintaining the logic of choice, which focuses on privatization and individualism, instead of the care that enhances network and co-responsibility.
\end{abstract}

Keywords: Mental Health, Narratives, Autonomy, Citizenship, Medication. 


\title{
Autonomía y Ejercicio de Derechos en la Experiencia de la Gestión Autónoma de la Medicación
}

\begin{abstract}
Resumen: El artículo investiga los efectos de uso de la Guía de Gestión Autónoma de la Medicación (GAM-BR), versión brasileña, en grupos de intervención en servicios de salud mental. El objetivo fue analizar narrativas de usuarios, psiquiatras y otros profesionales a partir de su relación con la prescripción de medicamentos, principio del trabajo con la Guía. Participaron en la investigación tres CAPS (Centros de atención psicosocial) del Sur del país, todos miembros de la investigación multicéntrica acerca de la GAM. Todo el material de audio fue grabado, transcrito y transformado en narrativas por medio de la extracción de los núcleos de significación. Se señala la expansión de la noción de autonomía de los usuarios y un mayor reconocimiento de sus derechos. Se presentaron dificultades para avanzar en el ejercicio de esos derechos, especialmente en cuanto al tratamiento farmacológico, que todavía se ve como una condición a la manutención del vínculo con los servicios. Es necesaria una mayor reflexión acerca del tema, considerando la manutención de la lógica de la elección, privatizante e individualista, en lugar de una lógica del cuidado que valorice el trabajo en la red y la corresponsabilización.
\end{abstract}

Palabras clave: Salud Mental, Narrativas, Autonomía, Ciudadanía, Medicación.

\section{Introdução}

A presente pesquisa dá continuidade a um estudo multicêntrico realizado por pesquisadores de universidades públicas brasileiras, integrantes da parceria internacional entre Brasil e Canadá, a qual teve como objetivo fomentar estudos sobre saúde mental e cidadania, formação de pesquisadores e transferência de tecnologia para a comunidade, tendo o protagonismo dos seus participantes como princípio (Marques, Palombini, Passos, \& Onocko, 2013; Passos, Otanari, Emerich, \& Guerini, 2013).

A primeira etapa desse estudo, iniciado em 2009, realizou quatro grupos de intervenção (GIs) em Centros de Atenção Psicossocial (CAPS) da região Sul e Sudeste do país. Ao longo de dois anos, os pesquisadores ocuparam-se de traduzir, adaptar e elaborar uma versão brasileira do Guia da Gestão Autônoma da Medicação Guia GAM, criado pelos serviços alternativos de saúde mental no Quebec, Canadá (Onocko-Campos et al., 2012). Esse guia é voltado às pessoas com transtornos mentais graves e pretende dar visibilidade à pluralidade de posições em face da medicação, afirmar o direito ao consentimento livre e esclarecido para utilização de psicofármacos, abordar a necessidade de compartilhar as decisões entre profissionais e usuários na ideia de que o tratamento em saúde mental é mais do que o uso de medicamentos, e que as pessoas são mais do que uma doença, não podendo ser reduzidas aos seus sintomas (Birman, 1992; Onocko-Campos et al., 2012; Torre \& Amarante, 2001).

Na segunda etapa da pesquisa, novos GIs foram realizados entre 2011 a 2014, sob a responsabilidade de três universidades brasileiras de diferentes estados (Sade, Barros, Melo, \& Passos, 2013). Neste artigo, apresentamos a experiência em um desses estados, em que os GIs aconteceram em três CAPS de municípios metropolitanos e os resultados foram colhidos a partir da construção das narrativas envolvendo a participação de usuários, trabalhadores e profissionais em formação.

\section{Método}

\section{Participantes}

Participaram da pesquisa na condição de prescritores de medicamentos: dois psiquiatras e um residente de psiquiatria no município A (referidos como A-P1, A-P2, A-P3, respectivamente); um psiquiatra no município B (B-P1). Dentre estes quatro, apenas um participou do GI (B-P1) respondendo às entrevistas em dois tempos: antes (tempo $=\mathrm{t}_{0}$ ) e depois (tempo $u m=t_{1}$ ) da participação no GI. Entre 
os outros três entrevistados, um respondeu à entrevista apenas em $\mathrm{t}_{0}$ (A-P3). Não houve participação de psiquiatras no município $\mathrm{C}$.

Outros profissionais, não prescritores de medicamentos, também participaram da pesquisa: um residente de saúde comunitária e um profissional do serviço no município A (A-D1, A-D2); e dois profissionais no município B (B-D1, B-D2). Dentre estes, dois estiveram presentes em $t_{0}$ e $t_{1}$ (A-D2, B-D1), e os outros dois em apenas um dos tempos (A-D1, B-D2). Todos participaram dos GIs com o Guia GAM. No município C, o GI foi protagonizado pelos usuários, sem a presença de profissionais.

Os grupos focais realizados em cada um dos CAPS participantes da pesquisa (nomeados, respectivamente, de GF-A, GF-B, GF-C) tiveram a seguinte participação de usuários integrantes dos GIs: no GF-A, seis usuários em $\mathrm{t}_{0}$ e dez usuários em $\mathrm{t}_{1}$; no GF-B, dez usuários em $t_{0}$ e seis usuários em $t_{1}$; no GF-C, seis usuários em T0 e nove usuários em $\mathrm{t}_{1}$.

\section{Instrumentos}

Foram realizados grupos focais e entrevistas com roteiros semiestruturados antes $\left(\mathrm{t}_{0}\right)$ e depois $\left(\mathrm{t}_{1}\right)$ dos GIs em cada um dos serviços participantes. Os grupos focais ocorreram com os usuários participantes dos GI; as entrevistas, com os psiquiatras que se dispuseram e, em relação aos profissionais não prescritores, com aqueles que participaram da realização dos GIs. Os roteiros utilizados foram específicos para cada um dos três segmentos, abordando os seguintes temas: autonomia, importância do contexto do usuário em seu tratamento, uso de medicamentos, direitos dos usuários da saúde mental.

\section{Procedimentos}

\section{Coleta de dados}

O áudio das falas produzidas nos grupos focais e nas entrevistas foi gravado e transcrito. As falas foram transformadas em narrativas a partir da extração de seus núcleos argumentais (Jorge et al., 2013; Onocko-Campos \& Furtado, 2008).

\section{Análise dos dados}

Construídas as narrativas, estas foram observadas à luz das semelhanças e discrepâncias a respeito das diferentes categorias temáticas que emergiram, levando em conta a divisão entre os três segmentos - psiquiatras, demais profissionais e usuários -, de acordo com a relação de cada um deles com a prescrição medicamentosa, mote do trabalho com o Guia GAM-BR. Na apresentação dos resultados e discussão, as referências diretas às narrativas são apresentadas entre aspas.

Havia a intenção de comparar o material colhido em $\mathrm{t}_{0}$ e $_{1}$; no entanto, devido a mudanças ocorridas nos serviços onde a pesquisa aconteceu - troca de profissionais na equipe ou descontinuidade da participação nos grupos -, não se priorizou tal intento, centrando a atenção nas similaridades e dessemelhanças encontradas no interior de um mesmo segmento ou de um segmento ou serviço a outro. Junto ao segmento dos usuários, fez-se possível uma análise temporal entre o momento anterior e o posterior ao grupo de intervenção.

\section{Considerações éticas}

Projeto de pesquisa aprovado pelo Comitê de Ética em Pesquisa (CEP) do Instituto de Psicologia da Universidade Federal do Rio Grande do Sul, sob o número de protocolo 21207, em 04 de julho de 2011.

\section{Resultados}

\section{Narrativas dos profissionais psiquiatras}

No que se refere à valoração do contexto de vida do usuário, houve entre os psiquiatras uma compreensão mais aproximada à sua prática após o GI. Expressões genéricas e impessoais como - "temos de respeitar a cultura do usuário" (A-P1/ $\left.\mathrm{t}_{0}\right)$ - deram lugar a afirmações referidas ao contexto da própria prática profissional - como "a inclusão do contexto de vida dos pacientes no atendimento acontece no exercício da minha prática, quando eu converso com o paciente, quando o escuto, quando vejo que ele está precisando e que eu posso contribuir". (B-P1/t $\mathrm{t}_{1}$.

Há certa similaridade na definição do que seria um usuário com autonomia, pensando-o como provido da capacidade de gerir a própria vida ("cuidar de questões do dia a dia"; "sair"; "decidir sobre o tratamento"; "cuidar das finanças"). Entretanto, diferem em relação à amplitude que conferem a esta capacidade. Um dos profissionais (A-P2) concebe como autônomo aquele que consegue cuidar da própria vida em todos os seus aspectos, citando questões referentes a alimentação, 
higiene, casa e circulação pela cidade; agrega ainda a capacidade, em alguma medida, de gestão financeira e medicamentosa. Outro (A-P1) afirma ser autônomo aquele que entende o seu tratamento e consegue administrá-lo. Enquanto a primeira narrativa relaciona autonomia a diversos aspectos da vida, a segunda relaciona-a apenas à administração do tratamento (reportando, paradoxalmente, a ideia de autonomia do usuário à obediência a uma prescrição).

Antes do GI, a hipótese de interrupção da medicação pelo usuário é vista por A-P2 com apreensão, de tal forma que o esforço é por encontrar uma estratégia que diminua os efeitos colaterais sem precisar interromper o uso de medicamentos. Quando a interrupção ocorre de fato, o psiquiatra não se vê como corresponsável pelo que poderá suceder ao usuário. Embora todos os entrevistados reconheçam o direito do usuário de interromper o tratamento medicamentoso, entendem que a suspensão desse tratamento torna-o mais vulnerável; além disso, de acordo com o relato dos psiquiatras, geralmente tal atitude configura abandono total do tratamento e do serviço.

Entre as narrativas, encontram-se alguns dos impasses em que se veem os médicos nesse processo: se, por um lado, "a função do médico não é obrigar ou policiar, apenas sugerir [a medicação]" (A-P2), sendo a escolha livre por parte do usuário, por outro, essa escolha pode ser condicionante da manutenção da relação com o médico - "se eles quiserem continuar se tratando comigo vão ter que tomar o medicamento, caso não queiram, não precisam tomar, mas também não precisam vir" (A-P2). A radicalidade dessa posição admite, porém, nuanças na relação

com aqueles pacientes que conseguem conversar a respeito - "olha eu não tô a fim de usar esse medicamento, mas não tem alguma outra coisa..."Nesses casos a gente tem uma possibilidade de diálogo e de estabelecer uma negociação a respeito de que tratamento o sujeito pode ou não fazer (A-P2).

Além disso, é referido que, na impossibilidade de se seguir um tratamento medicamentoso, poderá seguir vinculado a outras modalidades de tratamento:

É uma liberdade que o sujeito tem, querer ou não atendimento psiquiátrico. Se não quer o atendimento psiquiátrico, tranquilo, segue com o outro tratamento. Não impede ele de seguir com a psicoterapia, com a análise, com a terapia ocupacional, com o que quer que seja. A psiquiatria não é a única forma de tratamento (A-P2).

Tal afirmação contrasta com o entendimento de que a suspensão do tratamento medicamentoso resulta em abandono do serviço.

Após a intervenção, B-P1 refere tentar entender o motivo da recusa à medicação e pensar, junto à família e ao usuário, o que é possível ser feito. A responsabilidade é vista de forma compartilhada e é aceito que o usuário tem direito de decidir o que acha melhor para si, conhecendo as consequências e implicações de suas escolhas (A-P1). Entende-se que o limite das prescrições médicas é o sujeito em atendimento e que é este quem produzirá (ou não) demanda de tratamento para si. Permanece, porém, a crença em um saber especialista que não se coloca em questão nem pode ser substituído:

Nós levamos em conta o desejo do paciente, mas eu também tenho que levar em conta o saber acumulado da disciplina. Se ele voltar, terá que ser na condição de aceitar aquilo que o médico lhe propõe. Pode discutir, pode conversar, mas ele vai ter que aceitar. Se ele não aceitar aquilo que o médico propõe, não há qualquer condição (A-P2).

Nas situações de surto psicótico ou algum outro acontecimento que represente risco à integridade física de si ou de outrem, os psiquiatras entendem que a pessoa perde a liberdade de discussão e decisão. Apenas um deles (A-P3) diz considerar sempre a possibilidade de recusa ao uso de medicamentos por parte do usuário, citando a Lei Federal no 8.080/1990 (Brasil, 1990), ainda que esta não mencione a recusa do uso de medicamentos por parte do usuário. Considera, ainda, que o preocupante não é prescrever medicamentos contra a vontade do usuário, mas deixar de assisti-lo num momento em que se acredita que ele não pode decidir por si o que lhe é melhor (A-P3). Todos, nos dois tempos, reconhecem que há alternativas terapêuticas não medicamentosas; frisam, no entanto, o caráter indispensável da medicação para os casos graves.

Sobre a escolha medicamentosa, os psiquiatras dizem basear a decisão a partir do diagnóstico e dos principais sintomas apresentados, em combinação 
com as características pessoais do paciente. Um deles acrescenta a palavra como elemento a considerar:

Quando se prescreve um remédio, alimenta-se o paciente não somente com a pílula, mas também com palavras, e as palavras não têm um sentido único... Portanto, a prática clínica tem me mostrado que a prescrição do remédio não depende só da pílula, mas da palavra que dá suporte à pílula (A-P2).

Questionados sobre o saber dos usuários acerca dos medicamentos que utilizam, surgem duas posições divergentes. Um dos psiquiatras diz que "os usuários têm conhecimento principalmente em relação ao que sentem quando tomam os medicamentos; eles sabem muito sobre aquilo que eles tomam" (A-P1) - trata-se de um conhecimento pautado na experiência de uso, na experimentação dos efeitos positivos e colaterais. Em contrapartida, seu colega afirma que

a maioria dos pacientes sabe que precisa tomar o remédio, e poucos têm ideia de como funciona a medicação e de por que estão tomando. Isso tem a ver com a autonomia. Muitos pacientes não querem saber sobre a medicação que estão usando, do porquê estão usando, eles só querem melhorar. Querem que alguém tome a decisão por eles (A-P3)

Há o reconhecimento dos entrevistados quanto ao direito do usuário olhar e fazer cópia do prontuário, conversar sobre a medicação, informar se não tem condições de adquirir o medicamento - direitos que pode exercer enquanto estiver "bem, estável e tranquilo" (B-P1/t ${ }_{0}$. Os profissionais reconhecem, ainda, que ele terá o direito de recusar a medicação se estiver em condições de decidir isso, ou seja, "se não estiver em psicose, expondo-se moralmente ou ameaçando terceiros, circunstâncias em que seria necessária a internação compulsória" (B-P1/t $\mathrm{t}_{0}$. Participante do GI, B-P1, em $t_{1}$, refere desconhecer legislação específica sobre direitos dos usuários de saúde mental, ainda que tal legislação seja objeto de discussão no Guia GAM-BR.

Em relação à percepção dos efeitos do GI nos usuários, há diferenças nas manifestações dos psiquiatras. Uma das narrativas diz que não houve qualquer repercussão (A-P1). Em outra, aparecem as evidências de mudanças:

atualmente os usuários participantes do grupo GAM procuram os psiquiatras sem medo. A GAM tem mostrado resultado, pois os usuários começaram a ter uma comunicação melhor comigo, sentem-se mais à vontade para conversar e, assim, sinto que fiquei um pouco mais amigo deles. A minha participação no grupo contribuiu para isso, eu aprendi com a GAM, esse tipo de experiência foi inédito para mim (B-P1)

Cabe ressaltar que, enquanto B-P1, que destacou as diferenças produzidas nos usuários, havia participado do GI, A-P1, que não observou qualquer diferença, não teve contato direto com o GI - ainda que usuários sob seus cuidados participassem desse grupo. Isso parece indicar que, embora a estratégia GAM tenha fomentado maior consciência quanto a seus direitos nos usuários, esse aumento de consciência não chega a repercutir nos seus atendimentos com esses profissionais.

Como espaço para possíveis reclamações e sugestões acerca do serviço, os psiquiatras indicam as assembleias e associações de usuários ou, ainda, diretamente com o profissional implicado ou a coordenação do serviço. Esta posição permanece inalterada após a intervenção.

\section{Narrativas dos demais profissionais}

Os profissionais não prescritores entrevistados consideram importante para as estratégias de tratamento a análise do contexto social do usuário e referem construir os projetos terapêuticos singulares $(\mathrm{PTS})^{1}$ em conjunto com os usuários.

Antes dos GIs, autonomia foi definida por esses profissionais como independência e capacidade de fazer coisas por conta própria, constituída em relação com os outros e potencializada na medida da inserção no contexto social. Após a intervenção, definem-na como capacidade de gerir a própria vida, de acordo com seus valores, e entendem o reconhecimento dos direitos e dos valores pessoais e o seu exercício como potencializadora de autonomia.

A percepção dos profissionais quanto ao conhecimento dos usuários em relação aos medicamentos

${ }^{1}$ Cf. Silva, Sancho \& Figueiredo (2016). 
difere entre os serviços. Em B, os profissionais B-Dl e B-D2 foram enfáticos ao mencionar o pouco conhecimento dos usuários sobre os medicamentos que utilizam: "há um número grande de pessoas que não sabem nada sobre a sua medicação. Não têm conhecimento sobre o que usam e por que usam" (B-D2/ $\left.\mathrm{t}_{1}\right)$. A isso, atribuem um sentido:

muitas vezes, os pacientes não querem saber sobre suas medicações, como uma forma de negar a própria doença, para não pensar mais sobre isso. Outra situação é a de não querer ver a medicação como medicação. É quando se estabelece outro tipo de relação, de consumir todo e qualquer medicamento, não para ficar bem, não como tratamento. É outro tipo de relação; como uma fuga (B-D1/ $\left.\mathrm{t}_{1}\right)$.

Já no município A, apesar de mencionarem as dúvidas e o fato de que não são todos os usuários que se mostram com domínio de conhecimento quanto aos medicamentos que utilizam, parece haver uma valorização desse conhecimento, como refere A-D2 $\left(t_{0}\right)$ :

Os usuários têm algum conhecimento sobre os medicamentos como, por exemplo, o horário em que devem tomá-lo e o dia em que devem fazer a injeção de haldol novamente. Também já escutei deles algumas afirmações sobre o remédio ser forte ou fraco...

Em $t_{0}$ os trabalhadores afirmaram que esse conhecimento deve ser valorizado e pode ajudar a compreender melhor o usuário e discernir o que é efeito do medicamento e o que é da própria crise. B-D1 afirma que há, entre os usuários, muitas dúvidas em relação aos medicamentos e seus efeitos, e que "os pacientes não se sentem apropriados sobre esse tema para uma discussão com o médico". Já em $\mathrm{t}_{1}$, B-D1 vai considerar que a intervenção "trouxe o entendimento da relação de si com a medicação, dos motivos de usar determinado medicamento e não outro, da importância de falar ao médico como se sente e que ligações isso tem com a medicação".

Nas situações em que os usuários decidem interromper o uso de medicamento, a busca por entender o motivo é presente nas falas dos profissionais $\left(\mathrm{t}_{0} \mathrm{e} \mathrm{t}_{1}\right)$, assim como a preocupação em verificar se o usuário sabe quais são as possíveis consequências desse ato. Também entendem que é importante, nesses casos, acionar outros membros da equipe. A preocupação dos profissionais em relação às crises que a interrupção de medicamentos pode desencadear é constante nas respostas. Apenas A-D2 $\left(\mathrm{t}_{0}\right)$ apontou para um aspecto positivo dessa decisão:

se um usuário que eu acompanho decidisse parar de tomar medicamento, eu primeiramente suporia que ele está se sentindo bem, o que pode ser bom; mesmo assim, eu tentaria conversar sobre isso com ele e com o psiquiatra que lhe prescreveu a medicação.

Os trabalhadores destacam o cuidado com o usuário que decide parar de tomar a medicação; dizem que os casos são discutidos em equipe e que o desejo do usuário é respeitado, procurando alternativas como aumentar a frequência de sua presença no CAPS para acompanhar sua situação. Novamente, trata-se de um entendimento que contrasta com a associação feita pelos psiquiatras entre interrupção de tratamento medicamentoso e abandono do serviço. Porém, diferentemente dos psiquiatras, tanto em $\mathrm{t}_{0}$ como em $\mathrm{t}_{1}$, os demais profissionais consideraram recusar o tratamento medicamentoso como um direito do usuário, tendo sido citada a Carta dos Direitos dos Usuários do SUS (Brasil, 2013) por A-D2 $\left(\mathrm{t}_{0}\right)$. Alguns ponderaram, contudo, que esse direito depende da capacidade de discernimento do usuário e se ele coloca em risco a si ou a outros, mencionando a possibilidade de interná-lo compulsoriamente. A prescrição do medicamento contra a vontade do usuário é referida como uma experiência difícil, porém às vezes necessária. A-D1 diz que, "quando o usuário confia no profissional, a situação torna-se mais fácil".

Quando questionados sobre a contribuição do usuário no processo de tratamento, destaca-se a diferença em $t_{0}$ e $t_{1}$ que aparece na fala de A-D1. $E m \mathrm{t}_{0}$, esse profissional disse: "O usuário pode contribuir no seu processo de tratamento desde que se tenha feito um plano terapêtico em conjunto com ele e que ele se comprometa com isso". Já em $\mathrm{t}_{1}$, a sua resposta foi: "Penso que o usuário é o maior contribuinte em seu processo de tratamento". Nos dois momentos, a resposta é relacionada ao plano terapêutico do usuário, mas em $t_{1}$ fica evidente a importância do seu protagonismo. Há um deslocamento no modo de conceber o tratamento: antes referido como uma indicação a ser seguida, passa 
a ser descrito como sugerido e combinado em conjunto com o usuário, levando em conta a possibilidade deste de "aderir" à proposta e seu interesse em contribuir nessa construção.

A ideia de tratamento não medicamentoso se assemelha entre os profissionais. Em $t_{0}$, referiram como alternativas não medicamentosas os grupos e os atendimentos individuais. Em $t_{1}$, apresentaram possibilidades ampliadas, referindo a experiência em grupo, de poder falar e ter a quem falar, e de praticar exercícios como importantes estratégias de tratamento no CAPS. A-D1 enfatiza a importância do "estar no coletivo", enquanto A-D2 destaca singularidade e contexto do usuário como elementos para pensar recursos possíveis, alternativos à medicação.

Os espaços para reclamações e sugestões citados são assembleias realizadas no próprio serviço; ouvidoria; telefone de atendimento ao público de uma prefeitura municipal; secretaria de saúde; e grupos terapêuticos. Dois dos profissionais dizem aconselhar os usuários a primeiro conversar com o profissional alvo da crítica. Uma associação de usuários e familiares da rede de saúde mental do município B é citada em $t_{1}$ por B-D1.

A experiência de participação nos GIs é produtora de efeitos referidos pelos profissionais. No município B, apontou o quanto a vivência proporcionou mudanças na maneira de escutar e valorizar os direitos dos usuários: "O GAM me produziu isso: valorizar o direito do usuário em saúde mental. O contato com a pesquisa produziu uma diferença importante no meu modo de pensar" (B-D1/t 1 ). No município A, A-D1 mostra-se surpreendido pela capacidade dos usuários narrarem suas histórias; o impacto da intervenção neste serviço originou três grupos: um sobre cidadania, outro sobre histórias dos usuários e um terceiro, sobre medicamentos, proposto pelos psiquiatras.

\section{Narrativas dos usuários}

Em todos os grupos, os usuários afirmaram que podiam emitir opinião sobre seu tratamento, e a experiência com os GIs contribuiu para isso. "No CAPS, podemos conversar e opinar sobre o nosso tratamento com a equipe. A experiência do GAM ajudou bastante nessas conversas. Nós sentimos que os profissionais levam em conta a nossa opinião" (GF-C). Referiram que a experiência GAM permitiu-lhes aceder à noção de direitos - que desconheciam ou conheciam parcialmente - e assumir uma atitude mais ativa em relação ao seu tratamento - não somente um maior conhecimento sobre os medicamentos, mas a elaboração de estratégias para maior aceitação e apoio de pessoas próximas, reconhecendo a importância dos relacionamentos no adoecimento e no tratamento.

Porém o conhecimento adquirido acerca dos direitos e sobre os medicamentos é percebido ainda como parcial, não se traduzindo no seu exercício pleno. "Sabemos que temos direito à medicação e ao tratamento. Aconteceu, no CAPS, em alguns momentos, a discussão sobre o direito dos usuários, mas não sabemos todos eles e acreditamos que isso poderia ser mais falado" (GF-C). A experiência GAM não resultou numa ampliação ou modificação dos espaços e das formas oferecidas no serviço para tal - o que é condizente com a fala dos profissionais, que também apontam majoritariamente a assembleia geral como espaço precípuo de exercício de direito. Ainda que a experiência GAM tenha propiciado um aumento da participação dos usuários em relações dialógicas com os profissionais responsáveis pelo seu PTS e uma percepção mais crítica do serviço em que são atendidos, tal participação e percepção não se traduzem em processos de cogestão do PTS ou do próprio serviço. Além disso, o médico segue sendo o responsável exclusivo pela gestão do tratamento medicamentoso:

Nós estamos acostumados com a medicação, achamos bom tomá-la, pois precisamos dela. O GAM ajudou muito a fazer uma autoanálise, porque nós nem sabíamos que podíamos solicitar o prontuário pra ver o nosso acompanhamento médico, não sabíamos os códigos das doenças que temos. Estas portas foi o GAM que abriu para nós. Tanto sobre podermos falar nos grupos, como sobre o falar com o próprio médico. A psiquiatra nos ajudava tirando as dúvidas sobre os medicamentos (GF-B).

Mesmo para o grupo que afirmou, em $\mathrm{t}_{0}$, conhecer seus direitos de usuários, a decisão sobre a medicação aparece como de domínio exclusivo do especialista:

Nós temos direito enquanto usuários de saúde. Direito de não querer a medicação, mas também de aceitar o tratamento que é proposto no CAPS. Simplesmente ir contra um profissional que estudou, 
que sabe o que é melhor para as pessoas, pode ser teimosia. Teimosia é a pessoa achar que ela não precisa do medicamento, é ser orgulhosa, é próprio da cabeça das pessoas (GF-B).

Em $\mathrm{t}_{1}$, os usuários passam a reconhecer o direito de acesso ao tratamento e aos medicamentos, embora não cheguem a abordar o direito de recusa ao tratamento medicamentoso sem perda de acesso a outras formas de tratamento ou, se reconhecem esse direito, não se sentem convencidos, uma vez que entendem que a interrupção da medicação tem consequências graves para si:

Não temos certeza se podemos recusar o tratamento, a maioria de nós acredita que não. Uma de nós acredita que podemos, porém as consequências de não usar a medicação são muito graves, como já ocorreu com alguns de nós. E essas consequências estão sempre ligadas à internação. Quando paramos de usar os medicamentos, nos prejudicamos e prejudicamos a nossa família. Se quisermos, podemos vir ao CAPS sem ser nosso dia de atendimento e isso é muito bom, aqui ficamos mais tranquilos e é melhor do que apenas usar o medicamento (GF-C).

\section{Discussão}

As narrativas colhidas a partir das entrevistas com os profissionais dos serviços de saúde mental apontam para a predominância do saber especialista e técnico em detrimento do saber experiencial dos usuários, ainda que se perceba que este tem também seu lugar de importância. Embora, de forma geral, os profissionais mencionem o vínculo como tecnologia leve, referindo ao encontro singular com cada usuário, vigora uma concepção de cuidado bastante referenciada às tecnologias duras e leve-duras (ato de prescrever, indicação de internação compulsória, práticas terapêuticas grupais e individuais), que ganham primazia no tratamento (Merhy, 2002; Merhy \& Feuerwerker, 2009).

Assim, em relação ao direito de recusar a medicação, psiquiatras e demais profissionais ponderam a respeito com base na avaliação da capacidade de discernimento do usuário, se coloca ou não em risco a si e/ou aos outros, mencionando, conforme o caso, a possibilidade de internação compulsória.
Talvez, por sua condição de prescritores, os psiquiatras mostrem-se mais relutantes em aceitar a possibilidade de recusa de medicamentos por parte dos usuários e, ainda que não definam tal recusa como inviabilizadora do tratamento, entendem que ela leva ao abandono do serviço. Porém seria possível questionar se a interrupção do tratamento medicamentoso configura de fato uma atitude de abandono total do tratamento por parte do usuário. Dado um enunciado como: "não precisam tomar, mas também não precisam vir” (A-P2), é o usuário quem abandona o serviço ou os profissionais que o fazem abandonar? Ainda que tal enunciado se pretenda circunscrito ao tratamento psiquiátrico, sem incidência sobre as demais modalidades de tratamento, não deixa de colocar em causa o rompimento de um vínculo (Merhy, 2012).

Caberia ainda uma reflexão acerca da posição enunciada pelos profissionais, prescritores ou não, diante do usuário que recusa o tratamento medicamentoso, dadas as ambiguidades manifestas em tal posição. Via de regra, a decisão do usuário de suspender o uso de psicofármaco é vista com apreensão e mobiliza diversas atitudes: discussão do caso em equipe; respeito à decisão do usuário, buscando compreender seus motivos, condicionado à avaliação de seu grau de discernimento na ocasião; busca de outras alternativas medicamentosas (convencimento do usuário da necessidade de utilizá-lo); interrupção do acompanhamento psiquiátrico e abandono total do tratamento e do serviço (com a dubiedade já assinalada, em função do duplo sentido do genitivo em questão: o serviço é abandonado ou abandona?); interrupção do acompanhamento psiquiátrico com seguimento das outras modalidades de tratamento ofertadas pelo serviço, mantendo, porém, o tratamento farmacológico como indispensável nos casos graves; aumento da frequência ao serviço; internação compulsória em casos de surto psicótico com exposição moral e risco de agressão.

A disparidade dos procedimentos propostos acompanha percepções contrárias com respeito à responsabilidade pela tomada de decisão. Por um lado, há o reconhecimento de que o usuário tem o direito de decidir interromper o uso de medicamentos, com a ressalva, porém, de que o usuário em surto ou situação de risco não tem poder de decisão. Por outro lado, para uns, a responsabilidade 
pela interrupção do uso de medicamentos é do usuário; para outros, é compartilhada entre a equipe, o usuário e seus familiares.

As elaborações de Mol (2008), ao identificar duas lógicas - lógica da escolha e lógica do cuidado - que regem a abordagem ao usuário nas ações de saúde, ajudam-nos a compreender o que essa multiplicidade de atitudes, às vezes contraditórias entre si, coloca em causa. A lógica da escolha é perpassada por um ideal de liberdade individual, baseado na noção de razão esclarecida e de independência que permitiria a um sujeito bem informado decidir sozinho o que melhor lhe conviria. Tal perspectiva faz recair inteiramente no usuário a responsabilidade pelo ato de interrupção do tratamento medicamentoso. Como refere Barros (2015), "participar do próprio tratamento equivaleria a realizar escolhas individuais" (p. 246). A equipe profissional exime-se de tomar parte dessa decisão e de fazer-se responsável, junto com o usuário, das consequências advindas de tais escolhas. Já a lógica do cuidado entende o sujeito não como indivíduo "independente", mas referido a uma rede de relações que o constituem, da qual a equipe de saúde também vem a fazer parte. Em vez da responsabilização individual por uma escolha forçada entre alternativas já dadas de antemão (por exemplo, seguir ou não a prescrição medicamentosa), a lógica do cuidado propõe-se ao acompanhamento de um percurso em que novos problemas são inventados, bem como as alternativas para o seu enfrentamento, engajando, nesse processo, tanto quem cuida quanto quem é cuidado (Barros, 2015; Mol, 2008).

Percebe-se, assim, que a posição enunciada pelos profissionais diante do usuário que decide pela recusa do tratamento medicamentoso oscila entre essas duas lógicas: 1) a da escolha, que pressupõe um sujeito esclarecido, responsável individualmente por seus atos -condição da qual se vê excluído o sujeito em surto, por um lado, e, por outro, condição pela qual a equipe exime-se da responsabilidade pela continuidade do tratamento; 2) a do cuidado, que implica responsabilidades compartilhadas e construção coletiva - entre a equipe, o usuário e seus familiares - das alternativas possíveis de tratamento.

É curioso que, diante da pergunta acerca do seu direito de recusar o tratamento, os usuários se mostrem tão ou mais cautelosos que os profissionais. Talvez percebam o engodo em que se constitui o direito de escolha quando regido pela lógica da responsabilidade individual. Abster-se do tratamento medicamentoso torna-se um fardo pesado demais para carregar sozinho, implicando por vezes em uma internação não desejada. A referência à internação, por parte dos usuários, alude, por um lado, à ausência de outros dispositivos de acolhimento à crise que não o médico-hospitalar. Por outro lado, ao mencionar a internação como índice das graves consequências da interrupção do tratamento medicamentoso, os usuários incorporam a ideia, bastante presente no discurso e nas práticas dos profissionais, de que a medicação é parte imanente do tratamento nas situações de adoecimento psíquico grave. A recusa ao tratamento medicamentoso, embora reconhecida como direito, é vivida como desacato à autoridade do profissional ou como "teimosia". E, ainda, a associação imediata entre interrupção do tratamento medicamentoso e crise é indicativa de que outras modalidades de tratamento e de apoio comunitário não são apresentadas aos usuários como possibilidades alternativas à medicação para a sua estabilização - elas são complementares, mas não substitutas da medicação.

Com efeito, se as narrativas dos usuários apontam a GAM como estratégia efetiva de fomento ao seu protagonismo, auxiliando-os a melhor conhecerem seus direitos e a se posicionarem criticamente frente a seus tratamentos, isso não basta para provocar uma mudança no leque de ofertas terapêuticas e na forma das relações estabelecidas no serviço. Na maioria dos casos, a definição das terapêuticas oferecidas é prerrogativa dos profissionais, os quais delimitam também os espaços de reivindicação de direitos, circunscritos em geral às assembleias e às associações que reúnem usuários, familiares e trabalhadores - associações quase sempre vinculadas ao próprio serviço de saúde.

Por fim, e fazendo o necessário esforço de autoanálise, podemos nos perguntar sobre o que moveu a equipe de pesquisadores que inaugura o projeto multicêntrico a excluir da versão brasileira a parte que, no Guia original do Quebec, oferecia instruções, a partir de uma tomada de decisão do usuário, para reduzir progressivamente a quantidade de medicamentos ingerida, até a sua eliminação. A versão brasileira "deslocou o foco da retirada ou redução do remédio, para o da negociação, e a segunda parte do Guia foi reescrita, visando o incremento 
da participação do usuário na gestão do seu tratamento" (Onocko-Campos et al., 2012, p. 972, grifo dos autores).

As razões alegadas para tais adaptações apontam, de um lado, a posição manifesta pelos usuários participantes da etapa de elaboração do projeto de pesquisa de que o problema não era o direito de interromper o uso de medicamentos, mas o direito de ter acesso a eles; de outro, a escolha em realizar a pesquisa por dentro do Estado, isto é, no interior dos serviços de saúde mental vinculados ao Sistema Único de Saúde (SUS), implicando em negociação pela qual "concede-se ao Estado ao mesmo tempo em que se força o Estado a conceder" (Passos et al., 2013, p. 28). Mas qual seria a concessão em causa aqui?

Cede-se, como grupo de pesquisa, na afirmação a mais radical da possibilidade de o usuário decidir pela retirada dos medicamentos; e forçamos, ou pretendemos forçar, o Estado na figura dos seus agentes - profissionais e serviços de saúde mental a conceder o reconhecimento dos direitos dos usuários, a participação efetiva destes na formulação do seu projeto terapêutico, o exercício de cidadania no cotidiano dos serviços. Elementos presentes no ideário da reforma sanitária e do campo da saúde coletiva no Brasil, direitos, participação, cidadania forjam as ideias de autonomia e protagonismo - de trabalhadores e usuários - no SUS, em tensão com a função reguladora e de controle das populações pelo Estado. Contudo corremos inevitavelmente o risco das capturas. Assim, numa perspectiva neoliberal e privatizante da saúde, a ideia de protagonismo ou autonomia deságua na responsabilização individual do sujeito por seu adoecimento, bem como pelos cuidados à sua própria saúde. Ora, pautada na lógica do cuidado, conforme referido anteriormente, a decisão de interromper o uso de psicofármacos é uma responsabilidade compartilhada que requer acompanhamento profissional. Ao excluir de sua versão brasileira a segunda parte do Guia GAM original, poupamos os serviços e suas equipes de tomar contato com esta parte, livrando-os de ter de responder, coletivamente, à perspectiva de retirada dos medicamentos. Considerando o contexto da saúde mental no Brasil, havia a convicção de que a entrada nos serviços estaria inviabilizada se fosse mantida tal proposta. Contudo, ao tirar de cena as orientações para retirada progressiva da medicação, não estaríamos agindo também conforme a lógica da escolha, privatizante e individualista (se o usuário decide interromper o tratamento medicamentoso, então os profissionais não são responsáveis por isso e não têm o compromisso de seguir acompanhando-o nem de orientá-lo quanto ao modo de proceder para alcançar essa interrupção)?

\section{Considerações finais}

A pesquisa realizada aponta efeitos importantes na relação estabelecida entre usuários e trabalhadores dos CAPS participantes quanto ao reconhecimento dos direitos dos usuários no processo de seu tratamento a partir da experiência com o Guia GAM-BR. Segue, porém, sendo um desafio fazer com que o exercício desses direitos avance, especialmente no que se refere ao tratamento medicamentoso, aspecto considerado pelos envolvidos na pesquisa como condição para a manutenção do vínculo com os serviços e com os profissionais que os acompanham.

O investimento em espaços de formação com os trabalhadores dos serviços de saúde mostra-se, assim, fundamental para a problematização da manutenção de lógicas que a perspectiva da Reforma Psiquiátrica brasileira há muito vem questionando (Abrahao, Azevedo \& Gomes, 2017). Em especial, vemos como importante uma maior reflexão acerca da posição enunciada por profissionais (prescritores ou não) e mesmo por usuários diante da possibilidade de recusa do tratamento medicamentoso, tendo em vista a manutenção da lógica da escolha, privatizante e individualista, que esta posição parece sustentar.

A continuidade de pesquisas que acompanhem experiências de implantação de grupos GAM em diferentes serviços e contextos do campo da saúde, cuja intervenção se constitua também como uma possibilidade de Educação Permanente para os trabalhadores (Ceccim \& Ferla, 2009), é um dos desdobramentos que derivam do processo de investigação empreendido até o momento. Esperamos que possa fazer avançar as ações em saúde mental em direção à lógica do cuidado (Mol, 2008), valorizando cada vez mais o trabalho em rede e a corresponsabilização pelo cuidado entre quem cuida e quem é cuidado. 


\section{Referências}

Abrahao, A. L., Azevedo, F. F. M., \& Gomes, M. P. C. (2017). A produção do conhecimento em saúde mental e o processo de trabalho no Centro de Atenção Psicossocial. Trabalho, Educação e Saúde, 15(1), 55-71. http://dx.doi.org/ 10.1590/1981-7746-sol00041

Barros, L. M. R. (2015). A análise em uma pesquisa-intervenção participativa: O caso da Gestão Autônoma da Medicação (Tese de doutorado). Universidade Federal Fluminense, Niterói, RJ.

Birman, J. (1992). A cidadania tresloucada: Notas introdutórias sobre a cidadania dos doentes mentais. In B. Bezerra \& P. Amarante, Psiquiatria sem hospício: contribuições ao estudo da reforma psiquiátrica (pp. 71-90). Rio de Janeiro, RJ: Relume-Dumará.

Brasil. (1990). Lei $n^{\circ}$ 8.080, de 19 de setembro de 1990. Dispõe sobre as condições para promoção, proteção e recuperação da saúde, a organização e o funcionamento dos serviços correspondentes e dá outras providências. Brasília, DF: Presidência da República. http://www2.camara.leg.br/legin/fed/lei/1990/lei-8080-19-setembro -1990-365093-normaatualizada-pl.pdf

Brasil. (2013). Carta dos direitos dos usuários da saúde (4a. ed.). Brasília, DF: Ministério da Saúde.

Ceccim, R. B., \& Ferla, A. A. (2009). Educação permanente em saúde. In I. B. Pereira \& J. C. F. Lima (Orgs.), Dicionário da educação profissional em saúde (pp. 162-168). Rio de Janeiro, RJ: EPSJV.

Jorge, M. S. B., Onocko-Campos, R., Cavalcante, C.M., Vasconcelos, M. G. F., Lima, L. L., \& Carvalho, R. R. S. (2013). Concepções teóricas de análise de narrativas como foco na estratégia gestão autônoma da medicação (GAM) no contexto da pesquisa. In M. S. B. Jorge, R. M. Silva, \& A. M. F. Catrib (Orgs.), A transversalidade epistemológica da saúde coletiva: saberes e práticas (pp. 315-333). Fortaleza, CE: Eduece.

Marques, C.C., Palombini, A., Passos, E., \& Onocko, R.T.C. (2013). Sobre mudar de lugar e produzir diferenças: a voz dos usuários de serviços públicos de saúde mental. Mnemosine, 9(1), 106-126.

Mehry, E. E. (2002). Saúde: a cartografia do trabalho vivo. São Paulo, SP: Hucitec.

Merhy, E. E., \& Feuerwerker, L. C. M (2009). Novo olhar sobre as tecnologias de saúde: uma necessidade contemporânea. In A. C. S. Mandarino \& E. Gomberg (Orgs.), Leituras de novas tecnologias e saúde (pp. 29-74). São Cristóvão, SE: Editora UFS.

Merhy, E. E. (2012). Saúde e direitos: tensões de um SUS em disputa, molecularidades. Saúde e Sociedade, 21(2), 267-279. https://dx.doi.org/10.1590/S0104-12902012000200002

Mol, A. (2008). The logic of care. London: Routledge.

Onocko-Campos, R. T., \& Furtado, J. P. (2008). Narrativas: apontando alguns caminhos para sua utilização na pesquisa qualitativa em Saúde. In R. T. Onocko-Campos et al. (Orgs.), Pesquisa Avaliativa em Saúde Mental: desenho participativo e efeitos da narratividade (Vol. 1, pp. 321-334). São Paulo, SP: Aderaldo \& Rothschild Editores.

Onocko-Campos, R. T., Palombini, A. L., Silva, A. E., Passos, E., Leal, E. M., Serpa Júnior, O. D., \& Stefanello, S. (2012). Adaptação multicêntrica do guia para a gestão autônoma da medicação. Interface - Comunicação, Saúde, Educação, 16(43), 967-980. http://www.scielo.br/scielo.php?script=sci_arttext\& pid=S1414-32832012000400009\&lng=en\&tlng=pt

Onocko-Campos, R. T., Passos, E., Leal, E., Palombini, A. L., Serpa, O. D., Jr., Emerich, B. F., \& Carvalho, J. (Orgs.). (2012). Guia da gestão autônoma da medicação. Campinas, SP: Unicamp. https://www.fcm.unicamp.br/fcm/ sites/default/files/paganex/guia_gam_para_dowload_com_correcoes.pdf

Passos, E., Otanari, T. M. C., Emerich, B. F., \& Guerini, L. (2013). O comitê cidadão como estratégia cogestiva em uma pesquisa participativa no campo da saúde. Ciência e Saúde Coletiva, 18, 2919-2928. http://dx.doi.org/10.1590/ S1413-81232013001000016

Passos, E., Palombini, A. L., Onocko-Campos, R. T., Rodrigues, S. E., Melo, J., Maggi, P. M.; Marques, C. C.,Zanchet, L., Cervo, M. R., \& Emerich, B. (2013). Autonomia e cogestão na prática em saúde mental: o dispositivo da gestão autônoma da medicação (GAM). Aletheia, 41, 24-38.

Sade, C., Barros, L. M. R., Melo, J. J. M., \& Passos, E. (2013). O uso da entrevista na pesquisa-intervenção participativa em saúde mental: o dispositivo GAM como entrevista coletiva. Ciência \& Saúde Coletiva, 18(10), 2813-2824. https://dx.doi.org/10.1590/S1413-81232013001000006 
Silva, N. E. K., Sancho, L. G., \& Figueiredo, W. S. (2016). Entre fluxos e projetos terapêuticos: revisitando as noções de linha do cuidado em saúde e itinerários terapêuticos. Ciência \& Saúde Coletiva, 21(3), 843-852. https://dx.doi.org/ 10.1590/1413-81232015213.08572015

Torre, E. H. G., \& Amarante, P. (2001). Protagonismo e subjetividade: a construção coletiva no campo da saúde mental. Ciência \& Saúde Coletiva, 6(1), 73-85. http://dx.doi.org/10.1590/S1413-81232001000100006

Analice Palombini

Doutora em Saúde Coletiva pela Universidade Estadual do Rio de Janeiro. Docente do Instituto de Psicologia da Universidade Federal do Rio Grande do Sul, com atuação no curso de graduação em Psicologia e de Pós-Graduação em Psicologia Social e Institucional. Participa também da coordenação da Residência Integrada em Saúde Mental Coletiva, Porto Alegre - RS. Brasil.

E-mail: analice.palombini@ufrgs.br

D https://orcid.org/0000-0002-8332-8292

\section{Vera Pasini}

Doutora em Psicologia pela Pontifícia Universidade Católica do Rio Grande do Sul. Docente do Instituto de Psicologia da Universidade Federal do Rio Grande do Sul, com atuação no curso de graduação em Psicologia e na Residência Integrada em Saúde Mental Coletiva, Porto Alegre - RS. Brasil.

E-mail: verapasini@gmail.com

(iD) https://orcid.org/0000-0002-4579-8525

\section{Livia Zanchet}

Doutora em Psicologia Social e Institucional pela Universidade Federal do Rio Grande do Sul, em cotutela com a Université Côte D’Azur, Nice, França. Psicóloga nos serviços residenciais Foyers Les Baous em Vence, França.

E-mail: liviazanchet@gmail.com

(D) https://orcid.org/0000-0003-4603-2609

\section{Julia Bongiovanni}

Psicóloga do CAPS II Esteio. Mestre pelo Programa de Pós-Graduação em Psicologia Social e Institucional da Universidade Federal do Rio Grande do Sul, Porto Alegre - RS. Brasil.

E-mail: juliabongiovanni@yahoo.com.br

(D) https://orcid.org/0000-0002-3690-2001

\section{Marciana Zambillo}

Doutoranda no Programa de Pós-Graduação em Psicologia Social e Institucional da Universidade Federal do Rio Grande do Sul. Psicóloga no Hospital Universitário da Universidade Federal do Rio Grande. Porto Alegre - RS. Brasil. E-mail: marcianazambillo@gmail.com

(D) https://orcid.org/0000-0001-8302-6414

\section{Sandra Zanatta Guerra}

Especialista em Saúde Mental Coletiva pela Universidade Federal do Rio Grande do Sul. Psicóloga na Prefeitura Municipal de Gravataí. Porto Alegre - RS. Brasil.

E-mail: sandrazguerra@gmail.com

(i) https://orcid.org/0000-0002-8247-0852 


\section{Antonio B. Oliveira Neto}

Mestre pelo Programa de Pós-Graduação em Psicologia Social e Institucional da Universidade Federal do Rio Grande do Sul, Porto Alegre - RS. Brasil.

E-mail: antoniobolis@gmail.com

(1) https://orcid.org/0000-0002-8945-8741

\section{Letícia Presser Ehlers}

Especialista em Saúde Pública pela Escola de Saúde Pública do Estado do Rio Grande do Sul. Bacharela em Direito pela Pontifícia Universidade Católica do Rio Grande do Sul. Psicóloga na Prefeitura Municipal de Nova Santa Rita. Porto Alegre - RS. Brasil.

E-mail: leti_pe@hotmail.com

(D) https://orcid.org/0000-0001-9322-9004

Endereço para envio de correspondência:

Universidade Federal do Rio Grande do Sul, Instituto de Psicologia, Campus Saúde. Rua Ramiro Barcelos, 2.600, sala 136. CEP: 90035-003. Porto Alegre - RS. Brasil.

Recebido 19/01/2018

Aceito 19/03/2019

Received 19/01/2018

Approved 19/03/2019

Recibido 19/01/2018

Aceptado 19/03/2019

Como citar: Palombini, A., Pasini, V., Zanchet, L., Bongiovanni, J., Zambillo, M.; Guerra, S. Z., Oliveira Neto, A. B., \& Ehlers, L. P. (2020). Autonomia e Exercício de Direitos na Experiência da Gestão Autônoma da Medicação. Psicologia: Ciência e Profissão, 40, 1-13. https://doi.org/10.1590/1982-3703003190411

How to cite: Palombini, A., Pasini, V., Zanchet, L., Bongiovanni, J., Zambillo, M.; Guerra, S. Z., Oliveira Neto, A. B., \& Ehlers, L. P. (2020). Autonomy and Rights Exercise in Autonomous Management of Medication Experience. Psicologia: Ciência e Profissão, 40, 1-13. https://doi.org/10.1590/1982-3703003190411

Cómo citar: Palombini, A., Pasini, V., Zanchet, L., Bongiovanni, J., Zambillo, M.; Guerra, S. Z., Oliveira Neto, A. B., \& Ehlers, L. P. (2020). Autonomía y Ejercicio de Derechos en la Experiencia de la Gestión Autónoma de la Medicación. Psicologia: Ciência e Profissão, 40, 1-13. https://doi.org/10.1590/1982-3703003190411 